Pacific Journal of Mathematic 


\section{ON A THEOREM DUE TO SZ.-NAGY}

\section{R. S. PHILliPS}

B. Sz.-Nagy [4] has proved the following theorem:

THEOREM A. Let $\left[T_{t} ; t \geqq 0\right]$ be a strongly continuous semi-group of contraction operators on a Hilbert space $H$. Then there exists a group of unitary operators $\left[\mathbf{U}_{t},-\infty<t<\infty\right]$ on a larger Hilbert space $\mathbf{H}$ such that

$$
T_{t} y=\mathbf{P U}_{t} y,
$$

$y \in H, t \geqq 0$;

here $\mathbf{P}$ is the projection operator with range $H$. Then space $\mathbf{H}$ can be chosen in a minimal fashion so that $\left[\mathbf{U}_{t} H ;-\infty<t<\infty\right]$ spans $\mathbf{H}$. In this case $\left[\mathbf{U}_{t}\right]$ is strongly continuous and the structure $\left\{\mathbf{H}, \mathbf{U}_{t}, H\right\}$ is determined to within an isomorphism. ${ }^{1}$

The infinitesimal generator $L$ of the semi-group $\left[T_{t}\right]$ is defined by

$$
\lim _{\delta \rightarrow 0+} \delta^{-1}\left[T_{\delta} y-y\right]=L y
$$

for all $y \in H$ for which this limit exists. The operator $L$ is linear and closed with dense domain, $\mathfrak{D}(L)$ (see [1]). It is shown in [2] that $L$ is maximal dissipative in the sense that

$$
(y, L y)+(L y, y) \leqq 0,
$$

$y \in \mathfrak{D}(L)$,

and $L$ being maximal with respect to this property. Since $\left[\mathbf{U}_{t}\right]$ is a semi-group as well as a group of operators, the infinitesimal generator $\mathbf{L}$ of $\left[\mathbf{U}_{t}\right]$ also shares these properties; however in the case of a group of unitary operators $i \mathbf{L}$ is in addition self-adjoint.

The purpose of this note is to study the relation between $L$ and L. It turns out that $L$ is a restriction of $\mathbf{L}$ only when $L$ is maximal symmetric. In general $L$ is neither a restriction nor a projection of $\mathbf{L}$; in fact $\mathfrak{D}(\mathbf{L}) \cap H$ may contain only the zero element. Nevertheless we shall obtain $\mathbf{H}, \mathbf{L}$, and $\left[\mathbf{U}_{t}\right]$ directly from $L$, our principal tool being the discrete analogue of the above theorem, which is also due to Sz.-Nagy [4], namely

THEOREM B. Let $J$ be a contraction operator on a Hilbert space $H$. Then there exists a unitary operator $\mathbf{J}$ on a larger Hilbert space $\mathbf{H}$ such that

$$
J^{n} y=\mathbf{P J}^{n} y,
$$$$
y \in H, n \geqq 0 ;
$$

here $\mathbf{P}$ is the projection operator with range $H$. The space $\mathbf{H}$ can be

Received September 24, 1958. This paper was written under the sponsorship of the National Science Foundation, contract NSF G-4231.

1 Two structures $\left\{\mathbf{H}, \mathbf{U}_{t}, H\right\}$ and $\left\{\mathbf{H}^{\prime}, \mathbf{U}_{t}^{\prime}, H\right\}$ are isomorphic if there is a unitary map $\mathbf{V}$ of $\mathbf{H}$ onto $\mathbf{H}^{\prime}$ which is the identity on $H$ and is such that $\mathbf{V} \mathbf{U}_{t} y=\mathbf{U}_{t}^{\prime} \mathbf{V} y$ for all $y \in \mathbf{H}$. 
chosen in a minimal fashion in the sense that $\left[\mathbf{J}^{n} H ;-\infty<n<\infty\right]$ spans H. In this case the structure $\{\mathbf{H}, J, H\}$ is determined to within an isomorphism.

For a maximal dissipative operator $L$ with dense domain, it is shown in $[2, \S 1.1]$ that $(I-L)$ is one-to-one with range $\Re(I-L)=H$ and that

$$
J=(I+L)(I-L)^{-1}
$$

is a contraction operator with $\mathfrak{D}(J)=H$ and such that $(I+J)$ is one-toone. Applying Theorem $B$ we obtain the unitary operator $\mathbf{J}$ on the enlarged space $\mathbf{H}$ spanned by $\left[\mathbf{J}^{n} H ;-\infty<n<\infty\right]$ with $\mathbf{J}$ satisfying the property (4).

Lemma 1. The operator $(\mathbf{I}+\mathbf{J})$ is one-to-one.

Proof. Let $S$ be a contraction operator, set $3(S)=[y ; S y+y=\theta]$, and denote the projection operator with range $3(S)$ by $P_{S}$. Then the ergodic theorem (see [3, pp. 400-406]) asserts that

$$
\text { st. } \lim _{n \rightarrow \infty}(n+1)^{-1} \sum_{n=0}^{n}(-S)^{k}=P_{S}
$$

and that $S P_{S}=P_{S} S=-P_{S}$. We apply this result first to $J$ and then to J. Making use of (4) we see that

$$
\mathbf{P P}_{\mathrm{J}} y=P_{\mathrm{J}} y,
$$
$y \in H$.

As noted above $P_{J}=\Theta$, so that $\mathbf{P P}_{\mathbf{J}} \mathbf{P}=\Theta$. Actually $\mathbf{P}_{\mathbf{J}} \mathbf{P}=\Theta$; for otherwise there would exist a $y \in H$ with $\mathbf{P}_{\mathbf{J}} y \neq \theta$ so that

$$
\left(\mathbf{P P}_{\mathbf{J}} \mathbf{P} y, y\right)=\left(\mathbf{P}_{\mathbf{J}} y, y\right)=\left\|\mathbf{P}_{\mathbf{J}} y\right\|^{2}>0,
$$

which is impossible. Thus $\mathbf{P}_{\mathbf{J}} \mathbf{P}=\Theta$ and hence $3(\mathbf{J})$ is orthogonal to $H$. But this means that

$$
\mathbf{P}_{\mathbf{J}} \mathbf{J}^{n} H=\mathbf{J}^{n} \mathbf{P}_{\mathbf{J}} H=\theta,
$$

and we infer that $\mathbf{J}^{n} H$ is orthogonal to $3(\mathbf{J})$ for all $n$. The minimal property of $\mathbf{H}$ therefore requires that $3(\mathbf{J})=\theta$.

Remark. Associated with $\mathbf{J}$ is the resolution of the identity $[\mathbf{E}(\sigma)$; $-\pi<\sigma \leqq \pi]$ and the integral representation

$$
\mathbf{J}^{n}=\int_{-\pi}^{\pi} \exp (i n \sigma) d \mathbf{E}(\sigma) .
$$

Setting the restriction of $\mathbf{P E}(\sigma)$ to $H$ equal to $F(\sigma)$ we see by (4) that

$$
J^{n}=\int_{-\pi}^{\pi} \exp (i n \sigma) d F(\sigma) \text {. }
$$

The argument used in Lemma 1 applied to $S=\exp (i \mu) J$ shows that if 
$J$ has no eigenvalues of absolute value one, then neither does $\mathbf{J}$ and hence that both $\mathbf{E}(\sigma)$ and $F(\sigma)$ are strongly continuous in $\sigma$. Conversely, $F(\sigma)$ is strongly continuous then as is readily verified

$$
\begin{aligned}
(n & +1)^{-1} \sum_{k=0}^{n}[\exp (i \mu) \cdot J]^{k} y \\
& =\int_{-\pi}^{\pi} K_{n}(\sigma+\mu) d F(\sigma) y \rightarrow \theta,
\end{aligned}
$$

here

$$
K_{n}(\sigma)=(n+1)^{-1} \exp (i n \sigma / 2) \sin \left[\begin{array}{cc}
n+1 \\
2
\end{array}\right]\left[\begin{array}{ll}
\sin & \sigma \\
2
\end{array}\right]^{-1} .
$$

It then follows from the ergodic theorem that $3\{-\exp (i \mu) J\}=\theta$ and hence that $J$ has no eigenvalues of absolute value one.

Theorem. Set

$$
\mathbf{L}=(\mathbf{J}-\mathbf{I})(\mathbf{J}+\mathbf{I})^{-1} .
$$

Then L generates a strongly continuous group of unitary operators $\left[\mathbf{U}_{t} ;-\infty<t<\infty\right]$ such that

$$
T_{t} y=\mathbf{P U}_{t} y,
$$

$y \in H, t \geqq 0$

and $\left[\mathbf{U}_{t} H ;-\infty<t<\infty\right]$ spans $\mathbf{H}$.

Proof. It follows from the above lemma that $(\mathbf{I}+\mathbf{J})$ is one-to-one and hence that $\mathbf{L}$ is well-defined. Morever $\mathfrak{D}(\mathbf{L})=\mathfrak{R}(\mathbf{I}+\mathbf{J})$ is necessarily dense in $\mathbf{H}$ since otherwise $\left(\mathbf{I}+\mathbf{J}^{*}\right)$ would nullify some non-zero vector and since $\mathbf{J}^{-1}=\mathbf{J}^{*}$ the same would be true of $(\mathbf{I}+\mathbf{J})$. Further it is clear that $i \mathbf{L}$ is the Cayley tranform of $i \mathbf{J}$ and hence $\mathbf{L}$ generates a strongly continuous group of unitary operators which we shall denote by $\left[\mathbf{U}_{t}\right]$. In order to verify (7) we proceed to represent the resolvent $R(\lambda, L)=(\lambda I-L)^{-1}$ in terms of $J$ for $\lambda>0$. We see from (5) that

$$
y=2^{-1}(J u+u) \text { and } L y=2^{-1}(J u-u),
$$

$u \in H$.

Suppose next that $\lambda y-L y=f$. Replacing $y$ by $u$ as in (8) we obtain

$$
2^{-1} \lambda(J u+u)-2^{-1}(J u-u)=f
$$

so that

$$
u=2(1+\lambda)^{-1} \sum_{n=0}^{\infty}\left[(1-\lambda)(1+\lambda)^{-1}\right]^{n} J^{n} f, \quad \lambda>0 .
$$

Again making use of (8) we get

$$
y=2^{-1}(J u+u)=\sum_{n=0}^{n} a_{n}(\lambda) J^{n} f
$$

where 


$$
a_{0}(\lambda)=(1+\lambda)^{-1} \text { and } a_{n}(\lambda)=2(1-\lambda)^{n-1}(1+\lambda)^{-n-1} \text { for } n>0 .
$$

Thus $R(\lambda, L)$ can be represented by an absolutely convergent series in powers of $J$ for $\lambda>0$. Taking powers of $R(\lambda, L)$ we see that

$$
[R(y, L)]^{k}=\sum_{n=0}^{\infty} a_{n}^{(k)}(\lambda) J^{n},
$$

where again the series is absolutely convergent. Similarly

$$
\mathbf{R}(\lambda, \mathbf{L})^{k}=\sum_{n=0}^{\infty} a_{n}^{(k)}(\lambda) \mathbf{J}^{n},
$$

and it follows from (4) that

$$
[R(\lambda, L)]^{k} y=\mathbf{P}[\mathbf{R}(\lambda, \mathbf{L})]^{k} y, \quad y \in H, k \geqq 0, \lambda>0 .
$$

According to Yosdia's proof of the Hille-Yosida theorem (see [1]),

$$
T_{t}=\text { st. } \lim _{\lambda \rightarrow \infty} \exp \left(t B_{\lambda}\right) \text { and } \mathbf{U}_{t}=\underset{\lambda \rightarrow \infty}{\operatorname{st} . \lim _{\lambda \rightarrow \infty}} \exp \left(t \mathbf{B}_{\lambda}\right), \quad t \geqq 0 \text {, }
$$

where

$$
B_{\lambda}=\lambda^{2} R(\lambda, L)-\lambda I \text { and } \mathbf{B}_{\lambda}=\lambda^{2} R(\lambda, \mathbf{L})-\lambda \mathbf{I} .
$$

Thus for $y \in H$ the relation (9) implies

$$
\exp \left(t B_{\lambda}\right) y=\mathbf{P} \exp \left(t \mathbf{B}_{\lambda}\right) y, \quad y \in H, \lambda>0,
$$

and this together with (10) gives (7).

It remains to prove that $\mathbf{H}$ is the same as

$$
\mathbf{H}_{0}=\text { closed linear extension of }\left[\mathbf{U}_{t} H ;-\infty<t<\infty\right] \text {. }
$$

Let $\mathbf{P}_{0}$ be the projection of $\mathbf{H}$ onto $\mathbf{H}_{0}$. Then clearly $\mathbf{U}_{t} \mathbf{H}_{0} \subset \mathbf{H}_{0}$ for all real $t$, and since $\mathbf{U}_{t}{ }^{*}=\mathbf{U}_{-t}$ the same is true of the orthogonal complement to $\mathbf{H}_{0}$. As a consequence $\mathbf{P}_{0} \mathbf{U}_{t}=\mathbf{U}_{t} \mathbf{P}_{0}$ for all real $t$. Hence for $y \in \mathfrak{D}(\mathbf{L})$

$$
\mathbf{P}_{0} \mathbf{L} y=\lim _{\delta \rightarrow 0+} \delta^{-1}\left(\mathbf{P}_{0} \mathbf{U}_{\delta} y-\mathbf{P}_{0} y\right)=\lim _{\delta \rightarrow 0+} \delta^{-1}\left(\mathbf{U}_{\delta} \mathbf{P}_{0} y-\mathbf{P}_{0} y\right)=\mathbf{L} \mathbf{P}_{0} y .
$$

Thus $\mathbf{P}_{\mathbf{0}}$ commutes with $\mathbf{L}$ and hence with $\mathbf{J}$. But since $H$ is obviously contained in $\mathbf{H}_{0}$ we have

$$
\mathbf{J}^{n} H=\mathbf{J}^{n} \mathbf{P}_{0} H=\mathbf{P}_{0} \mathbf{J}^{n} H \subset \mathbf{H}_{0} .
$$

The minimal property of $\mathbf{H}$ asserted in Theorem $\mathrm{B}$ therefore implies that $\mathbf{H}=\mathbf{H}_{0}$. This concludes the proof of the theorem.

It should be noted that since $i \mathbf{L}$ is self-adjoint, the largest restriction to $H$ of $i \mathbf{L}$ will be symmetric. On the other hand if $i L$ is symmetric then it is easily verified that $J$ is an isometry and hence that $\mathbf{J}$ is an extension of $J$; in this case then $\mathbf{L}$ will be an extension of $L$. However in general if $u \in H$ and $y=\mathbf{J} u+u$, then $z=\mathbf{P} y=J u+u \in \mathfrak{D}(L)$ 
and $L \mathbf{P} y=\mathbf{P L} y$; each $z \in \mathfrak{D}(L)$ can be so represented. A simple example shows that $\mathfrak{D}(L) \cap H$ may contain only the zero element. ${ }^{2}$

\section{REFERENCES}

1. E. Hille and R. S. Phillips, Functional analysis and semi-groups, Amer. Math. Soc. Coll. Publ., XXXI (1957).

2. R. S. Phillips, Dissipative operators and hyperbolic systems of partial differential equations, Trans. Amer. Math. Soc., (1959).

3. F. Riesz and B. Sz.-Nagy, Leşons d'analyse fonctionnelle, 3rd edition Budapest (1955).

4. B. Sz.-Nagy, Transformations de l'espace de Hilbert. fonctions de type positif sur un groupe, Acta Sci. Math. Szeged, 15 (1954) 104-114.

THE UNIVERSITY OF CALIFORNIA,

LOS ANGELES

2 Suppose $H$ is one-dimensional and $T_{t}=\exp (-t)$. The Sz.-Nagy construction for $\mathbf{H}$ in Theorem $\mathrm{B}$ then results in $\mathbf{H}=l_{2}$, the space of complex-valued sequences $y=\left\{\eta_{n}\right.$; $-\infty<n<\infty\}$ with

$$
(y, z)=\sum_{n=-\infty}^{\infty} \bar{\eta}_{n} \bar{\xi}_{n},
$$

$\mathbf{J}\left\{\eta_{n}\right\}=\left\{\eta_{n-1}\right\}$, and $\mathbf{P}\left\{\eta_{n}\right\}=\left\{\eta_{n}^{\prime}\right\}\left(\eta_{0}^{\prime}=\eta_{0} ; \eta_{n}^{\prime}=0\right.$ for $\left.n \neq 0\right)$. Then relation (8) as applied to $\mathbf{J}$ and $\mathbf{L}$ asserts that for each $\left\{\eta_{n}\right\} \in \mathbb{D}(\mathbf{L})$ there is a $\left\{\mu_{n}\right\} \in \mathbf{H}$ such that

$$
2 \eta_{n}=\mu_{n-1}+\mu_{n}, \quad 2\left[\mathrm{~L}\left\{\eta_{n}\right\}\right]_{n}=\mu_{n-1}-\mu_{n} .
$$

If we also require that $\left\{\eta_{n}\right\} \in H$, then $\mu_{n-1}+\mu_{n}=0$ for all $n \neq 0$ and this together with the condition $\sum\left|\mu_{n}\right|^{2}<\infty$ implies that $\mu_{n}=0$ for all $n$. It follows that $\mathfrak{D}(L) \cap H=\theta$. 



\section{PACIFIC JOURNAL OF MATHEMATICS}

\section{EDITORS}

\section{David Gilbarg}

Stanford University

Stanford, California

R. A. Beaumont

University of Washington

Seattle 5 , Washington
A. L. Whiteman

University of Southern California

Los Angeles 7, California

L. J. PAIGE

University of California

Los Angeles 24, California

\section{ASSOCIATE EDITORS}
E. F. BECKENBACH
C. E. BURGESS
E. HEWITT
A. HORN

\author{
V. GANAPATHY IYER \\ R. D. JAMES \\ M. S. KNEBELMAN \\ L. NACHBIN
}
I. NIVEN
E. G. STRAUS
T. G. OSTROM
H. L. ROYDEN
G. SZEKERES
M. M. SCHIFFER
F. WOLF
K. YOSIDA

\section{SUPPORTING INSTITUTIONS}

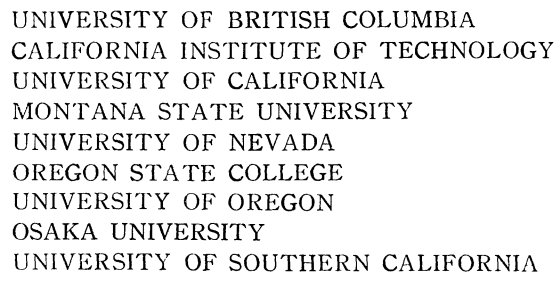

UNIVERSITY OF BRITISH COLUMBIA CALIFORNIA INSTITUTE OF TECHNOLOGY UNIVERSITY OF CALIFORNIA MONTANA STATE UNIVERSITY UNIVERSITY OF NEVADA OREGON STATE COLLEGE UNIVERSITY OF OREGON OSAKA UNIVERSITY UNIVERSITY OF SOUTHERN CALIFORNIA

STANFORD UNIVERSITY

UNIVERSITY OF TOKYO

UNIVERSITY OF UTAH

WASHINGTON STATE COLLEGE

UNIVERSITY OF WASHINGTON

AMERICAN MATHEMATICAL SOCIETY CALIFORNIA RESEARCH CORPORATION HUGHES AIRCRAFT COMPANY SPACE TECHNOLOGY LABORATORIES

Printed in Japan by Kokusai Bunken Insatsusha (International Academic Printing Co., Ltd.), Tokyo, Japan 


\section{Pacific Journal of Mathematics}

\section{Vol. 9, No. 1 \\ May, 1959}

Julius Rubin Blum and Murray Rosenblatt, On the structure of infinitely

divisible distributions . ............................. 1

Robert Geroge Buschman, Asymptotic expressions for

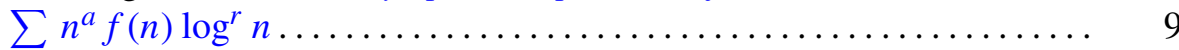

Eckford Cohen, A class of residue systems $(\bmod r)$ and related arithmetical

functions. I. A generalization of Möbius inversion .............. 13

Paul F. Conrad, Non-abelian ordered groups ................... 25

Richard Henry Crowell, On the van Kampen theorem............... 43

Irving Leonard Glicksberg, Convolution semigroups of measures ........ 51

Seymour Goldberg, Linear operators and their conjugates ............ 69

Olof Hanner, Mean play of sums of positional games .............. 81

Erhard Heinz, On one-to-one harmonic mappings ................ 101

John Rolfe Isbell, On finite-dimensional uniform spaces . . ........... 107

Erwin Kreyszig and John Todd, On the radius of univalence of the function

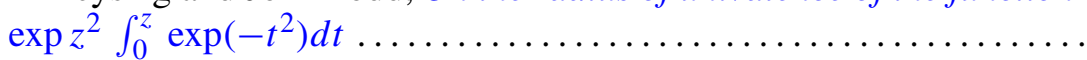

Roger Conant Lyndon, An interpolation theorem in the predicate

calculus......................................... 129

Roger Conant Lyndon, Properties preserved under homomorphism ........ 143

Roger Conant Lyndon, Properties preserved in subdirect products ....... 155

Robert Osserman, A lemma on analytic curves ................ 165

R. S. Phillips, On a theorem due to Sz.-Nagy..................... 169

Richard Scott Pierce, A generalization of atomic Boolean algebras ....... 175

J. B. Roberts, Analytic continuation of meromorphic functions in valued fields................................. 183

Walter Rudin, Idempotent measures on Abelian groups ................ 195

M. Schiffer, Fredholm eigen values of multiply-connected domains ........ 211

V. N. Singh, A note on the computation of Alder's polynomials ......... 271

Maurice Sion, On integration of 1-forms ...................... 277

Elbert A. Walker, Subdirect sums and infinite Abelian groups........... 287

John W. Woll, Homogeneous stochastic processes . .................. 293 